\title{
Piecewise Polynomial Fitting with Trend Item Removal and Its Application in a Cab Vibration Test
}

\author{
Wu Ren $\left(\mathbb{D},{ }^{1}\right.$ Qiongqiong Ren, ${ }^{1}$ Lin Han, ${ }^{1}$ Ying Liu, ${ }^{2}$ and Bo Peng ${ }^{3}$ \\ ${ }^{1}$ School of Biomedical Engineering, Key Lab of Neurosense and Control, Xinxiang Key Laboratory of Biomedical Information \\ Research, Xinxiang Medical University, Xinxiang, Henan 453003, China \\ ${ }^{2}$ The First Affiliated Hospital of Xinxiang Medical University, Xinxiang, Henan 453100, China \\ ${ }^{3}$ Hunan Special Equipment Inspection and Testing Research Institute Zhangjiajie branch, Zhangjiajie, Hunan 427000, China
}

Correspondence should be addressed to Wu Ren; renwu88@126.com

Received 7 November 2017; Revised 27 February 2018; Accepted 3 March 2018; Published 12 April 2018

Academic Editor: Giuseppe Maruccio

Copyright (C) $2018 \mathrm{Wu}$ Ren et al. This is an open access article distributed under the Creative Commons Attribution License, which permits unrestricted use, distribution, and reproduction in any medium, provided the original work is properly cited.

\begin{abstract}
The trend item of a long-term vibration signal is difficult to remove. This paper proposes a piecewise integration method to remove trend items. Examples of direct integration without trend item removal, global integration after piecewise polynomial fitting with trend item removal, and direct integration after piecewise polynomial fitting with trend item removal were simulated. The results showed that direct integration of the fitted piecewise polynomial provided greater acceleration and displacement precision than the other two integration methods. A vibration test was then performed on a special equipment cab. The results indicated that direct integration by piecewise polynomial fitting with trend item removal was highly consistent with the measured signal data. However, the direct integration method without trend item removal resulted in signal distortion. The proposed method can help with frequency domain analysis of vibration signals and modal parameter identification for such equipment.
\end{abstract}

\section{Introduction}

Cab vibration has an important influence on both the driver and the machine. In actual tests, the general acceleration signal is complex. Thus, research on the acceleration vibration signal is of great theoretical and practical significance [1-3]. Liu et al. [4] proposed a low-frequency signal removal method for the FFT transform to achieve a better integration effect. Cai and Wang [5] proposed the empirical mode decomposition method (EMD) to remove trend items and improve the effect of frequency domain integration. Ribeiro et al. $[6,7]$ proposed a fast Fourier transform digital double integration (FFT-DDI) method for dealing with lowfrequency vibration signals and applied it to vibration signals collected by integrated circuit piezoelectric (ICP) sensors to obtain more accurate integration results.

Good results have been obtained by integrating the short-term acceleration signal to remove the trend item. This provides a method for processing long-term acceleration vibration signals [8]. This paper proposes a method of piecewise fitting with trend item removal for a better integration effect. The low-frequency component of a signal plays a decisive role in the magnitude of the velocity vibration amplitude. In a cab vibration test, a long-period zero drift trend item is usually present and is caused by various changes in the equipment and instruments. This can completely alter the calculation results [9-11]. In addition, the signal collection may be subject to other external frequencies for vibration interference, so the collected data contain useless interference. Thus, data filtering is essential [12]. Sarrouy [13] has proposed a numerical method based on polynomial chaos expansions to process stochastic eigenvalue problems efficiently. Several random distributions had been tested in the end. Dufan et al. [14] had used piecewise polynomial fitting to fit the low-energy image to the high-energy image. And the result was constrained by its distance to the fitted image. In the end, the ASD-POCSbased optimization schedule has been proposed and the numerical simulations were taken to verify the algorithm then. A sequential testing procedure to distinguish between 
a piecewise polynomial trend superimposed by short-range dependence and that imposed by long-range dependence had been examined by Baek [15]. Finally, the proposed method was illustrated to the historical Northern Hemisphere temperature data. A novel constrained fit of the boundary curve which benefits from the smoothness of spline approximation and the computational efficiency of the linear program had been developed by Daouia et al. [16]. In the end, the utility of this method was illustrated through applications to some real data sets. And the simulation result was also presented to show its superiority to the best-known methods. A new denoising procedure which enhances the Raman information whilst reducing unwanted contributions from the most frequent noise sources was presented by González-Vidal et al. [17]. The method relied on cubic penalized spline fitting and mathematical morphology and required no user input. A novel level set method named as polynomial piecewise constant approximation (PPCA) to well segment images with serious intensity inhomogeneity had been proposed by Min et al. [18]. Finally, the visual and experimental results demonstrated the proposed method can yield better results than the existing classical local models for segmenting images. The research of cab vibration is mainly as follows: Deboli et al. [19] had done the whole body vibration measurement of horizontal and vertical transmissibility of an agricultural tractor seat. Finally, they had drawn the conclusion that the rolling and pitching effects can be reduced by using specific suspension systems along the horizontal and lateral directions. Humphreys et al. have investigated the system dynamics which included the human operator and biodynamic feedthrough. Then, two methods of compensation for biodynamic feedthrough based on their models had been developed. The results showed that the operators perform better and prefer the vibration-compensating controllers over their nonvibration-compensating counterparts [20]. Sim et al. [21] had proposed a hydropneumatic suspension applied with the semiactive control method. Then, the ride comfort has been predicted by using the vehicle model based on computer simulation in the end. Mahmoodabadi et al. [22] have introduced a novel combination of the particle swarm optimization (PSO) and the genetic algorithm (GA). The hybrid algorithm uses the operators such as mutation, traditional or classical crossover, multiple crossovers, and PSO formula. At the end, the algorithm had been used for the Pareto optimal design of a five-degree of freedom vehicle vibration model. The comparison of the obtained results demonstrated it has a good result in the work. Lepine et al. [23] had proposed to use machine learning to identify shocks present in acceleration signals measured on road vehicles. Next, a machine learning algorithm has been trained to identify shocks buried within road vehicle vibration signals. The results showed that the machine learning algorithm was considerably more accurate and reliable in identifying shocks than the more common approaches. Burdzik [24] had presented a novel method for research on exposure to nonlinear vibration of passenger car suspension as a nonlinear dynamical system. The advantage of this method is the possibilities of the precise analysis of chosen frequency bands. Then, the influence of damping of the shock absorber on exposure to vibration had been discussed. Probabilistic metrics were used for a robust Pareto multiobjective optimum design of the five-degree of freedom vehicle vibration model by Jamali et al. [25]. The robustness of the design obtained using such a probabilistic approach was shown and compared with that of the design obtained using the deterministic approach in the end.

Until now, methods with trend item removal have usually employed polynomial fitting and EMD. Based on previous studies, this paper proposes a more accurate vibration signal processing method called piecewise integration with trend item removal. This can help with the research on the vibration characteristics of a driver and personal comfort.

When the EMD and polynomial fitting methods are used to remove a vibration signal trend item, there is usually a large error at the start and the end of the signal [26]. Piecewise integration can be used to solve this problem. An overlapping method is proposed for the segmentation of a signal. As shown in Figure 1(a), the collected signal A1B3 is divided into three parts: A1B1, A2B2, and A3B3. Each data part is represented by dn which is the length of data. Then, the segmented data are processed separately. The middles of the start and end sections of each data segment are taken as data during the trend item removal procedure. As shown in Figure 1, A1C1, C1C2, and C2B3 should be considered the processed data. Data overlap can also be observed before and after the segmentation points $\mathrm{C} 1$ and $\mathrm{C} 2$. Suppose that the overlap radio is $m$; then, the number of these data pieces is $\mathrm{dn} \cdot m$. The benefit of this segmentation method is that relatively large head-to-tail errors can be removed. Suppose that the length of the vibration signal is $n$; the quotient $p$ can be obtained by $n / \operatorname{dn}(1-2 \mathrm{~m})$, and the remainder is $q$. If $q$ is larger than $2 \mathrm{dn} \cdot m$, the signal is divided into $p+1 \mathrm{sec}-$ tions. Otherwise, it is divided into $p$ sections. In both cases, the last section is taken as the remaining data. As can be seen in Figures 1(b)-1(d), the signal processed by the EMD method and polynomial fitting method has a major error because the vibration has deviated from the 0 level. And the signal processed by segmentation piecewise polynomial fitting with the removing trend term is closed to the actual data.

Based on the above method, the acceleration vibration signal can be segmented by the conventional trend item removal process. Furthermore, the velocity and displacement can be obtained from the integral. In the operation, the following two cases can be obtained: the individual integral after trend item removal and the integral merged back into the whole data segment after trend item removal [27]. After the signal segmentation and trend item removal, the integral values before and after the section points may differ. In this case, the average integral value near the section points can be used to replace the separated ones. Next, a three-order spline curve correction is carried out at several points near the section to ensure the maximum smoothness of the segmented connection.

The trend item removal method and various integration results can be compared through an example. Suppose the 


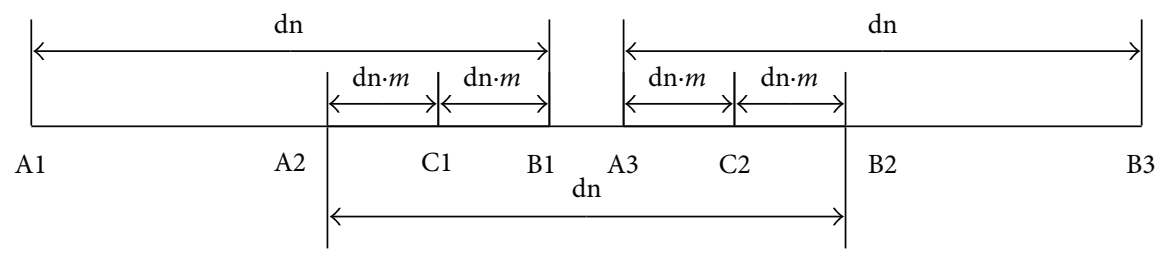

(a)

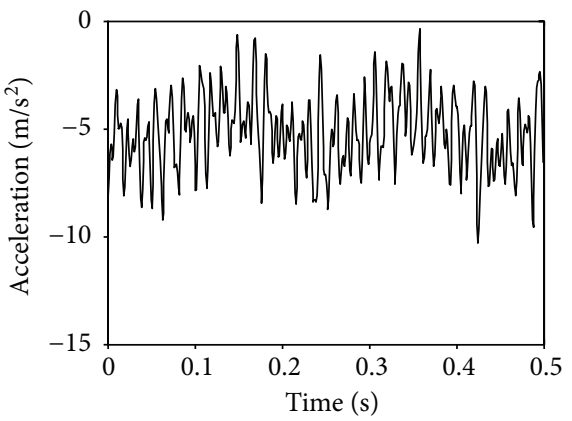

(b)

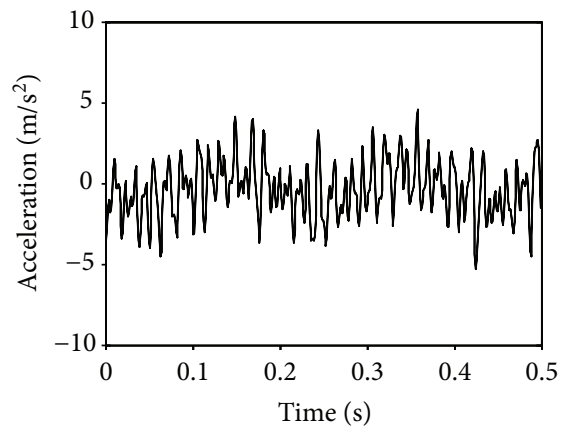

(c)

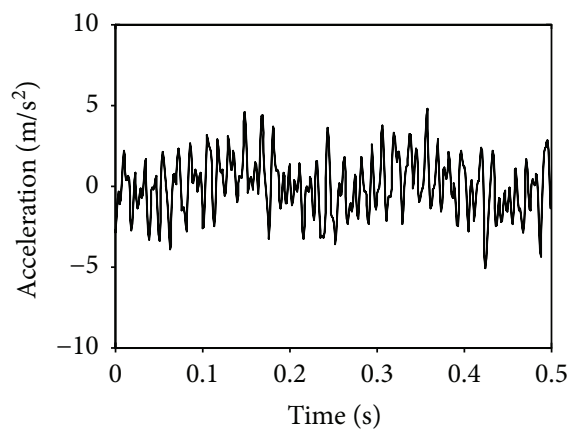

(d)

FIGURE 1: Signal segmentation and method comparison. (a) Signal segmentation. (b) EMD method. (c) Polynomial fitting method. (d) Segmentation piecewise polynomial method.

vibration acceleration signal containing the trend item is given as follows:

$$
\begin{aligned}
a(t)= & -\sum_{i=1}^{6} \sin \left(2 \pi f_{i} t+\varphi_{i}\right)+\operatorname{hann}(t) \\
& +t(t-20)(t-40)(t-60)+7,
\end{aligned}
$$

where $f_{i}$ is the component frequencies: $5,52,64,80,96$, and $158 \mathrm{~Hz} ; \varphi_{i}$ is the component initial phase angles: $10^{\circ}, 20^{\circ}$, $30^{\circ}, 40^{\circ}, 150^{\circ}$, and $70^{\circ}$; hann is the Hann function; and $t$ is the time.

In (1), the acceleration signal contains six sine curves with amplitudes of 1 and one integrated trend item. The integrated trend item consists of three parts: a Hann trend item, a polynomial trend item, and a DC (direct current) component. Suppose that the sampling frequency fs is $1000 \mathrm{~Hz}$, the signal sampling time is $60 \mathrm{~s}$, and the length $n$ of the signal is 60,000 . Figure 2 compares the actual acceleration signal and the signal containing the trend item, and Figure 3 shows the local contrast details.

\section{Acceleration Piecewise Integral with Removing Trend Item}

Three integral methods have been researched in this section.

2.1. Direct Integral without Removing Trend Item. The velocity data can be obtained from the original acceleration signal integral. To compare the results with and without trend item removal, the acceleration signal was not processed. Figure 4 shows the result. The velocity demonstrated a significant shift. If further integration is performed, a displacement with more distortion data will be obtained. This proves that trend item removal is indispensable to vibration signal processing.

2.2. Global Integral after Piecewise Polynomial Fitting with Removing Trend Term Trend. The entire acceleration signal was divided into 2000 segments with an overlap ratio of $10 \%$. In each segment, the three-order polynomial was used to fit the trend item. Next, the velocity signal was calculated by combining the segmentation of the acceleration signal with trend item removal. The order of the velocity trend item polynomial was four. Figure 5 shows the acceleration curve, 


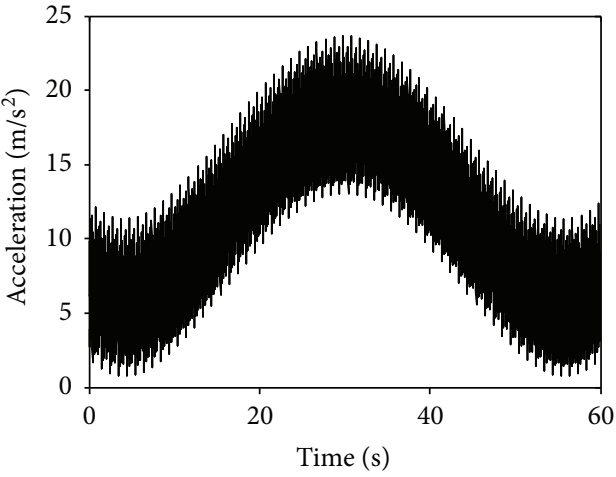

(a)

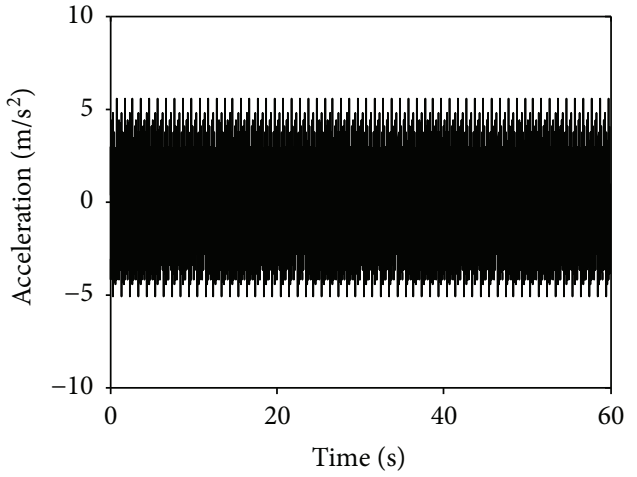

(b)

FIGURE 2: Signal comparison between acceleration containing trend item and actual acceleration. (a) $0 \sim 60 \mathrm{~s}$ acceleration containing trend item. (b) $0 \sim 60 \mathrm{~s}$ actual acceleration.

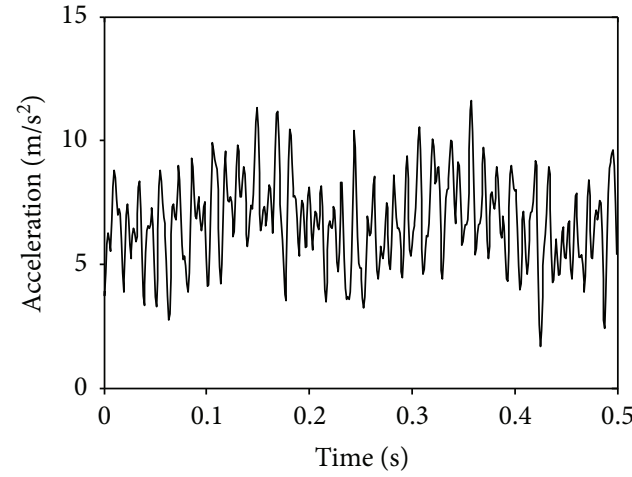

(a)

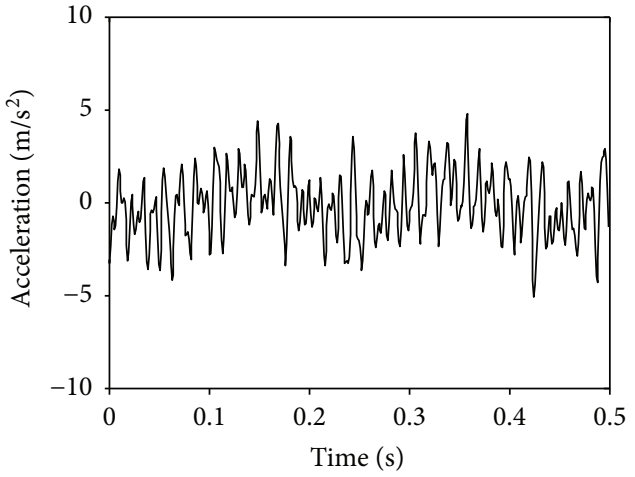

(b)

FIGURE 3: Signal local comparison between acceleration containing trend item and actual one. (a) $0 \sim 0.5 \mathrm{~s}$ acceleration containing trend item. (b) $0 \sim 0.5 \mathrm{~s}$ actual acceleration.

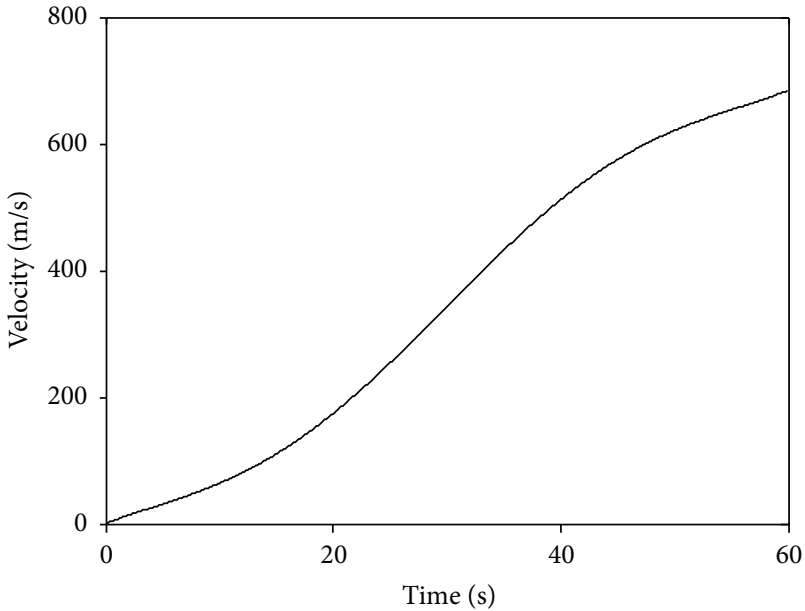

FIGURE 4: Velocity curve by acceleration integral without removing trend item.

and Figure 6 shows the curve of the velocity signal after trend item removal. When the actual acceleration and velocity signals were compared, the following parameters were calculated: $R_{\mathrm{a}}=0.9972, D_{\mathrm{a}}=4687.8, R_{\mathrm{v}}=0.9605$, and $D_{\mathrm{v}}=254.5793$ ( $R$ is the geometric parallelism of two sets of data, and $D$ is the geometrical distance of two sets of signals. They can be used to evaluate that one method is good or not). As shown in Figures 5 and 6, the results were better when the trend items were removed for both the acceleration and the velocity.

2.3. Direct Integral after Piecewise Polynomial Fitting with Removing Trend Item. The entire acceleration signal was divided into 2000 segments with an overlap ratio of $10 \%$. The order of the polynomial fitted to the acceleration signal was three. Then, the direct integral was obtained by removing the trend item in each segment. The velocity trend item polynomial order was four. Next, the segmented velocity signals were combined into an integrated one, as shown in Figure 7(a). Figure 7(b) shows the detailed velocity signal in the first $0.5 \mathrm{~s}$. When compared with $R_{\mathrm{v}}$ and $D_{\mathrm{v}}$ for the actual signal of 0.9965 and 62.1394, respectively, the parameters calculated with the integration method were closest to the actual signal. Thus, the validity of this method was verified. This method can also be used to obtain the displacement from integrating the velocity signal. The order of the polynomial fitted to the displacement signal was five. Figure 8(a) shows the displacement signal after trend item removal. Figure 8(b) shows the detailed displacement signal in the first $0.5 \mathrm{~s} . R_{\mathrm{v}}$ and $D_{\mathrm{v}}$ for the actual signal were 0.9930 and 2.4091, respectively. 


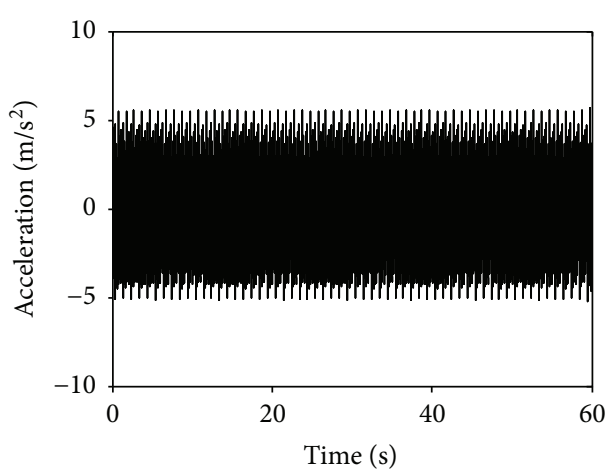

(a)

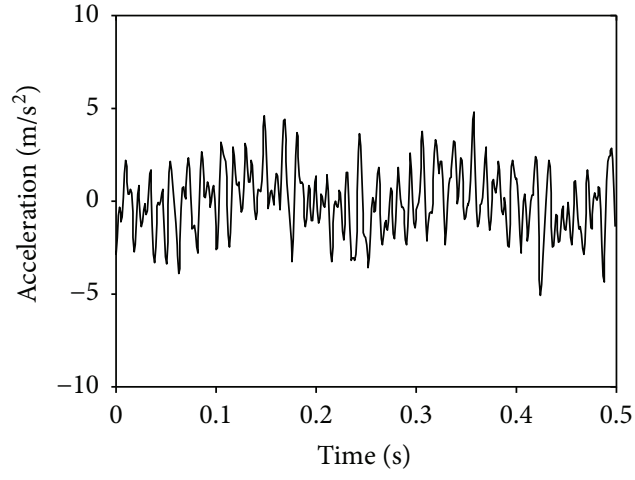

(b)

FIGURE 5: Acceleration of piecewise polynomial fitting with removing trend term. (a) $0 \sim 60 \mathrm{~s}$ acceleration of piecewise polynomial fitting with removing trend term. (b) $0 \sim 0.5 \mathrm{~s}$ acceleration of piecewise polynomial fitting with removing trend term.

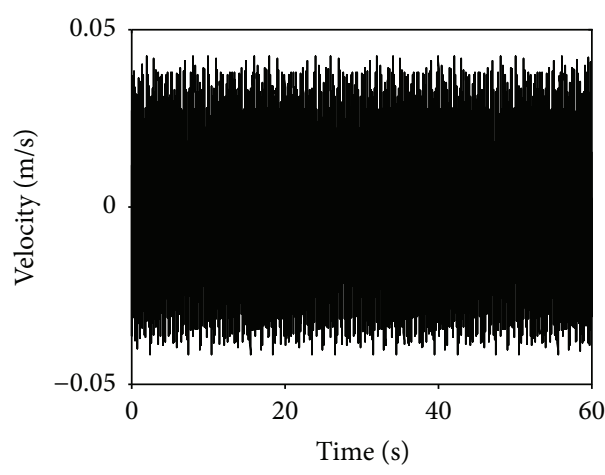

(a)

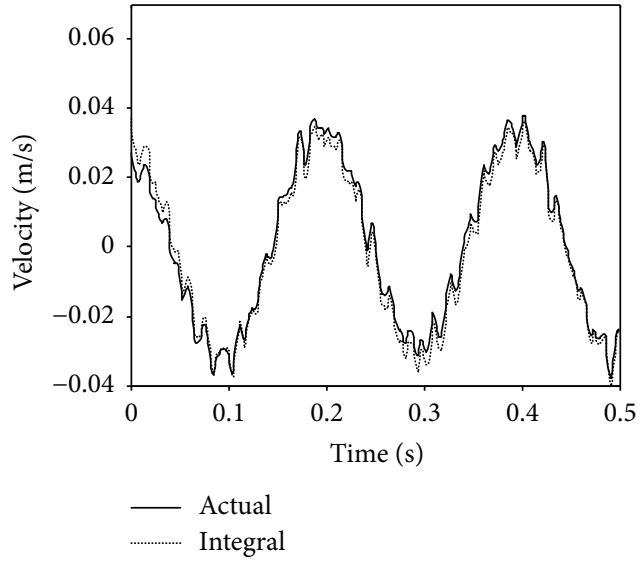

(b)

FIGURE 6: Global integral velocity of piecewise polynomial fitting with removing trend term. (a) $0 \sim 60$ s global integral velocity with removing trend term. (b) $0 \sim 0.5 \mathrm{~s}$ global integral velocity with removing trend term.

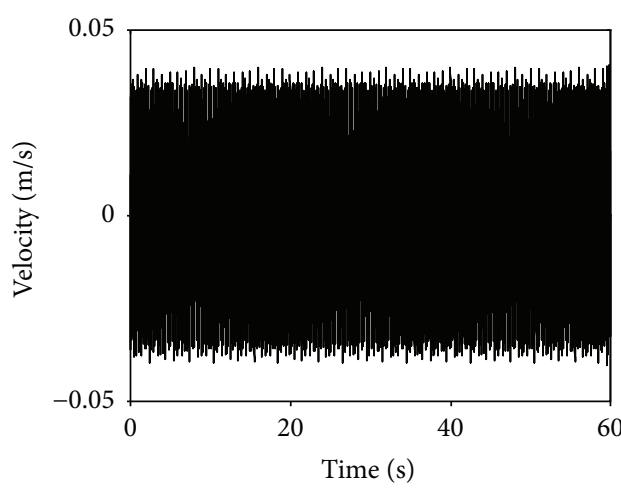

(a)

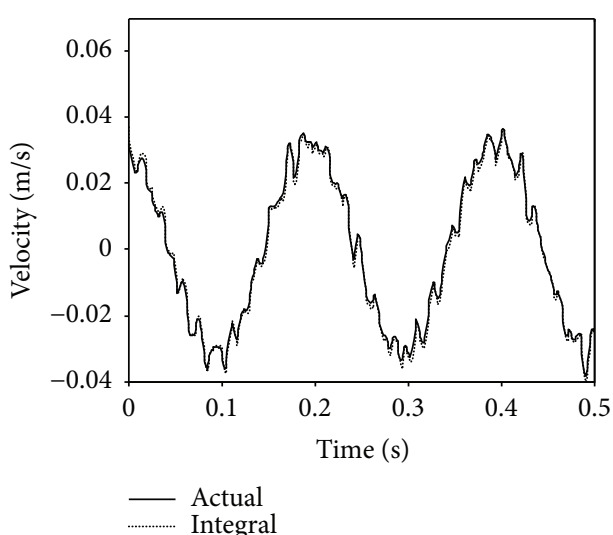

(b)

FIGURE 7: Direct integral velocity of piecewise polynomial fitting with removing trend term. (a) $0 \sim 60$ s direct integral velocity with removing trend term. (b) $0 \sim 0.5 \mathrm{~s}$ direct integral velocity local contrast.

There was a larger error before $0.1 \mathrm{~s}$, but the integration results were basically consistent with the actual value after $0.1 \mathrm{~s}$. This also explains why there was a greater error in the signal start section with trend item removal. In practical applications, therefore, the beginning of the data should be avoided. 


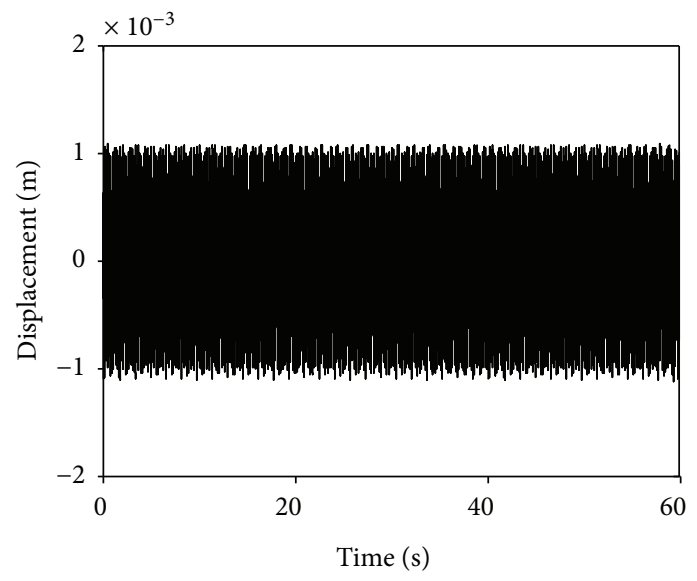

(a)

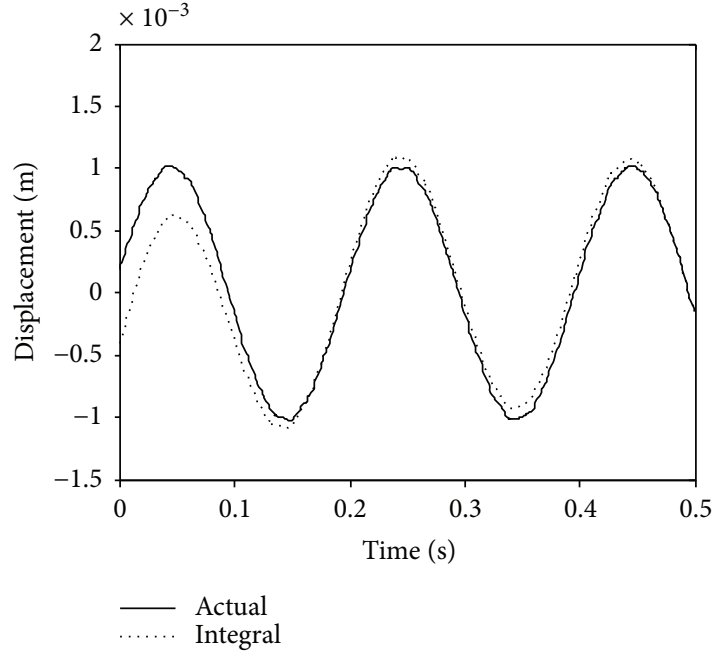

(b)

FIGURE 8: Direct integral displacement of piecewise polynomial fitting with removing trend term. (a) $0 \sim 60$ s direct integral displacement with removing trend term. (b) $0 \sim 0.5 \mathrm{~s}$ direct integral displacement with removing trend term.

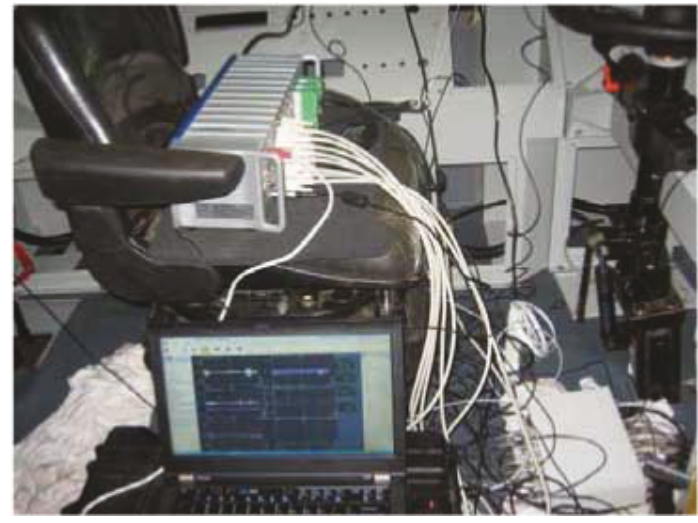

Figure 9: Cab vibration test.

The above example showed that, when the vibration signal lasts for a long time, the trend item removal of the whole signal cannot obtain a good result. The piecewise integration method reduces the overall EMD decomposition time and polynomial fitting order, which makes the numerical calculation faster and stable. A comparison of the results showed that direct integration of subsegments provided a better performance than global integration. The polynomial fitting method is relatively simple for trend item removal after integration. Furthermore, the calculation results are ideal. Therefore, piecewise polynomial fitting with trend item removal is a feasible method for the integration of an acceleration signal.

\section{Piecewise Polynomial Fitting with Removing Trend Term Integral Application in a Cab Vibration Signal Processing}

An ICP acceleration sensor and DS-NET multichannel data collection instruments were used to measure the cab vibration of a metal transfer vehicle. Figure 9 shows the vibration test.

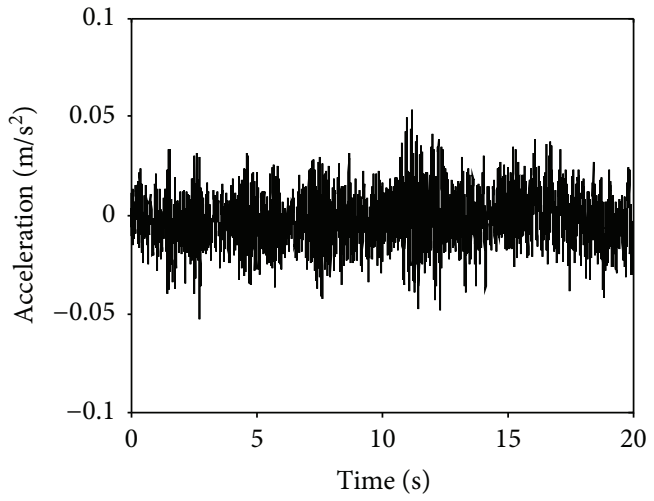

Figure 10: The measured acceleration of the cab dashboard.

The acceleration signal at the cab dashboard was taken as the original data. The sampling frequency was $1000 \mathrm{~Hz}$, and the sampling time was $20 \mathrm{~s}$ as shown in Figure 10. The measured acceleration signal indicated a certain drift. So it has vibration characteristic. If the acceleration signal in Figure 10 is directly integrated without the removal of the trend item, the calculated velocity and displacement signals would be distorted, as shown in Figure 11. In Figure 11(a), we can see that the velocity gained with integrated direction did not have the vibration characteristic, and in Figure 11(b), the displacement signal curve direction integrated by acceleration is smooth. The vibration characteristics of the velocity and displacement both basically disappear, which is contrary to the basic theory of vibration and is wrong. The piecewise polynomial fitting with the removal of the trend item from the integral was then adopted. Figure 12 shows the velocity and displacement curves. The velocity and displacement curve were improved, and the feasibility of the method has been proved. As seen in Figure 12(a), the velocity signal obtained by the piecewise polynomial fitting with the removal of the trend item integrate which used the same acceleration data in Figure 10 


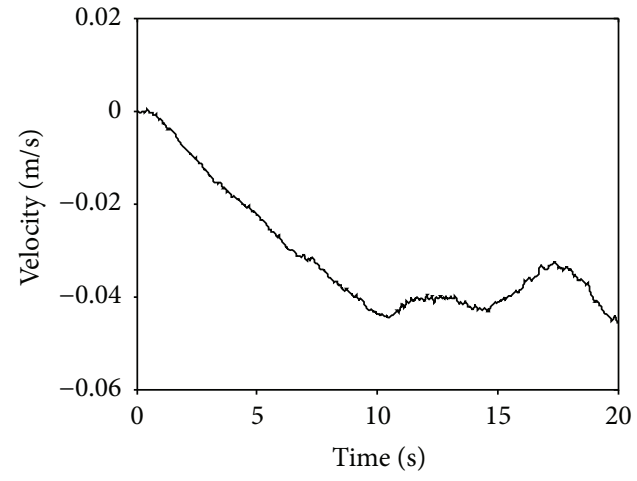

(a)

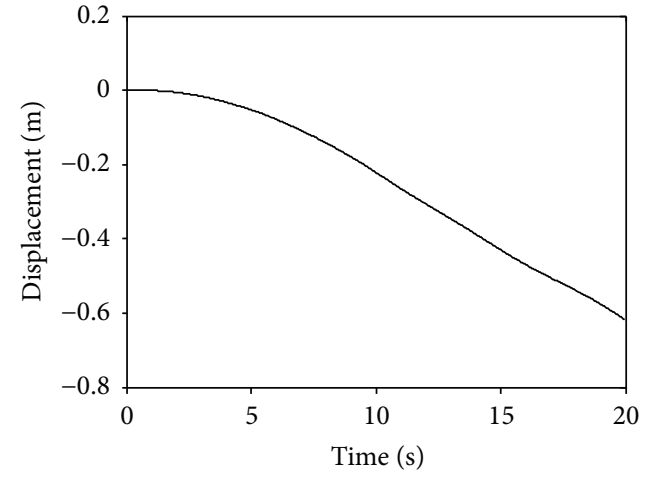

(b)

FIGURE 11: Velocity and displacement by acceleration direct integral without removing trend item. (a) Velocity by acceleration direct integral. (b) Displacement by acceleration direct integral.

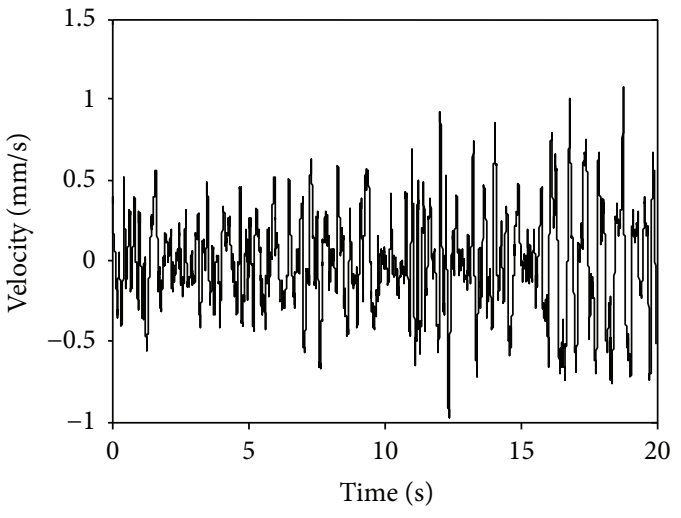

(a)

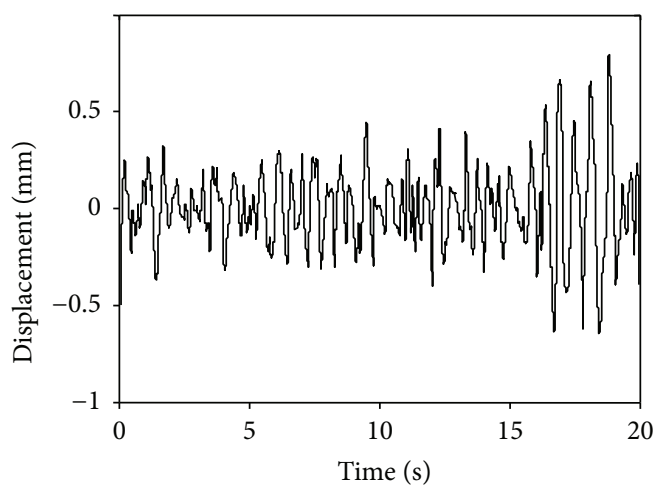

(b)

FIGURE 12: Velocity and displacement by piecewise polynomial fitting with removing trend item of acceleration integral. (a) Velocity by piecewise polynomial fitting with removing trend item. (b) Displacement by piecewise polynomial fitting with removing trend item.

has vibration characteristic. Meanwhile, the displacement signal obtained by the piecewise polynomial fitting with the removal of the trend item integrate method also has vibration characteristic as the acceleration data. By using the proposed method, we can get the velocity and displacement data by the acceleration signal without doing the actual experiment because the acceleration signal usually can be measured easier than the other two signals. This can cut the cost and time of the experiment.

\section{Conclusion}

This paper proposes a method for trend item removal by polynomial fitting in vibration signal processing. The effects and characteristics were evaluated. A segmentation method was developed to calculate the velocity and displacement by integrating the acceleration. Direct integration without trend item removal, global integration after piecewise polynomial fitting with trend item removal, and direct integration after piecewise polynomial fitting with trend item removal were evaluated. The simulation examples showed that direct integration after piecewise polynomial fitting with trend item removal provided the results closest to expectations. Finally, a vibration test was performed on a special equipment cab. The velocity and displacement signals were obtained by integrating the actual collected acceleration signal, which proved the feasibility of the piecewise integration method.

\section{Conflicts of Interest}

The authors declare that they have no conflicts of interest.

\section{Acknowledgments}

This work was supported by "Human machine dynamic characteristics of multi degree of freedom rehabilitation displacement device" (172102310542), "Multi-DOF rehabilitation patient transfer device dynamics characteristic and human-machine safety comfort research" (505140), "Human comfort study by elevator transportation based on multibody dynamics" (no. 2015KYJH33), the Natural Science Foundation of Henan Province (152102310357), and Study on behavioral evaluation of human lower limb (Development Project of School of Biomedical Engineering, Xinxiang Medical University (2018-BME-KFKT-05)). 


\section{References}

[1] S. S. Rao and F. F. Yap, Mechanical Vibrations, Prentice hall, Upper saddle river, 2011.

[2] S. Xinhui, Research on Frequency Domain Modal Parameter Identification and Its Software Implementation, Nanjing university of aeronautics and astronautics, Nanjing, China, 2010.

[3] Z. Jian, Mechanical Fault Diagnosis Technology, Machinery industry press, Beijing, 2008.

[4] L. Jicheng, X. Qinghua, and Z. Jianxin, "Method of vibration displacement measured with acceleration sensor," Xiandai leida(Modern radar), vol. 29, no. 5, pp. 69-71, 2007.

[5] C. Feng and W. Dayuan, "Acceleration integration transformation based on EMD," Journal of Scientific Instrument, vol. 07 , no. 1, pp. 12-13, 2007.

[6] J. G. T. Ribeiro, J. T. P. de Castro, and J. L. F. Freire, "New improvements in the digital double integration filtering method to measure displacements using accelerometers," in Proceedings-SPIE the International Society for Optical Engineering, vol. 1no. 1, pp. 538-542, Orlando, FL, USA, 2001.

[7] J. G. T. Ribeiro, J. T. P. de Castro, and J. L. F. Freire, "Using the FFT-DDI method to measure displacements with piezoelectric,resistive and ICP accelerometers," in Conference and exposition on structural dynamics, vol. 4no. 6, pp. 19-26, Kissimee, FL, USA, 2003.

[8] H. Van der Auweraer, P. Guillaume, P. Verboven, and S. Vanlanduit, "Application of a fast-stabilizing frequency domain parameter estimation method," Journal of Dynamic Systems, Measurement, and Control, vol. 123, no. 4, pp. 651658, 2001

[9] B. Peeters, H. Van Der Auweraer, P. Guillaume, and J. Leuridan, "The PolyMAX frequency-domain method: a new standard for modal parameter estimation," Shock and Vibration, vol. 11, no. 3-4, 409 pages, 2004.

[10] B. Cauberghe, "Applied frequency-domain system identification in the field of experimental and operational modal analysis," Faculteit toegepaste wetenschappen, vol. 38, no. 5, pp. 247-259, 2004.

[11] H. Luo, MATLAB GUI Design Study Notes[M], Beijing university of aeronautics and astronautics press, Beijing, 2011.

[12] B. Peeters, G. Lowet, H. Van der Auweraer, and J. Leuridan, "A new procedure for modal parameter estimation," Sound and Vibration, vol. 38, no. 1, pp. 24-29, 2004.

[13] E. Sarrouy, O. Dessombz, and J. J. Sinou, "Piecewise polynomial chaos expansion with an application to brake squeal of a linear brake system," Journal of Sound and Vibration, vol. 332, no. 3, pp. 577-594, 2013.

[14] W. Dufan, Z. Li, L. Li, S. Le, and Y. Xing, "Prior image guided under sampled dual energy reconstruction with piecewise polynomial function constraint," Computational and Mathematical Methods in Medicine, vol. 2013, Article ID 437917, 7 pages, 2013.

[15] C. Baek, "A piecewise polynomial trend against long range dependence," Journal of the Korean Statistical Society, vol. 44, no. 3, pp. 457-468, 2015.

[16] A. Daouia, H. Noh, and B. U. Park, "Data envelope fitting with constrained polynomial splines," Journal of the Royal Statistical Society: Series B (Statistical Methodology), vol. 78, no. 1, pp. 3-30, 2016.

[17] J. J. González-Vidal, R. Pérez-Pueyo, and M. J. Soneira, “Automatic morphology-based cubic p-spline fitting methodology for smoothing and baseline-removal of Raman spectra," Journal of Raman Spectroscopy, vol. 48, no. 6, pp. 878-883, 2017.

[18] H. Min, W. Jia, X. F. Wang, Y. Zhao, and Y. T. Luo, "A polynomial piecewise constant approximation method based on dual constraint relaxation for segmenting images with intensity inhomogeneity," Pattern Recognition, vol. 73, no. 7, pp. 15-32, 2018.

[19] R. Deboli, A. Calvo, and C. Preti, "Whole-body vibration: measurement of horizontal and vertical transmissibility of an agricultural tractor seat," International Journal of Industrial Ergonomics., vol. 58, no. 3, pp. 69-78, 2017.

[20] H. C. Humphreys, W. J. Book, and K. M. Feigh, "Development of controller-based compensation for biodynamic feedthrough in a backhoe," Proceedings of the Institution of Mechanical Engineers, Part I: Journal of Systems and Control Engineering., vol. 228, no. 2, pp. 107-120, 2014.

[21] K. Sim, H. Lee, J. W. Yoon, C. Choi, and S. H. Hwang, "Effectiveness evaluation of hydro-pneumatic and semi-active cab suspension for the improvement of ride comfort of agricultural tractors," Journal of Terramechanics, vol. 69, no. 2, pp. 23-32, 2017.

[22] M. J. Mahmoodabadi, A. A. Safaie, A. Bagheri, and N. Nariman-Zadeh, "A novel combination of particle swarm optimization and genetic algorithm for Pareto optimal design of a five-degree of freedom vehicle vibration model," Applied Soft Computing., vol. 13, no. 5, pp. 2577-2591, 2013.

[23] J. Lepine, V. Rouillard, and M. Sek, "On the use of machine learning to detect shocks in road vehicle vibration signals," Packaging Technology and Science, vol. 30, no. 8, pp. 387398, 2017.

[24] R. Burdzik, "Novel method for research on exposure to nonlinear vibration transferred by suspension of vehicle," International Journal of Non-Linear Mechanics, vol. 91, no. 5, pp. 170-180, 2017.

[25] A. Jamali, M. Salehpour, and N. Nariman-Zadeh, "Robust Pareto active suspension design for vehicle vibration model with probabilistic uncertain parameters," Multibody System Dynamics, vol. 30, no. 3, pp. 265-285, 2013.

[26] C. Devriendt, G. De Sitter, and P. Guillaume, “An operational modal analysis approach based on parametrically identified multivariable transmissibilities," Mechanical Systems and Signal Processing, vol. 24, no. 5, pp. 1250-1259, 2010.

[27] J. Honggang, C. Wu, and C. Bin, "Based on RF curve fitting algorithm frequency range modality analysis," Metrology \& measurement technique, vol. 17, no. 10, pp. 8-11, 2011. 


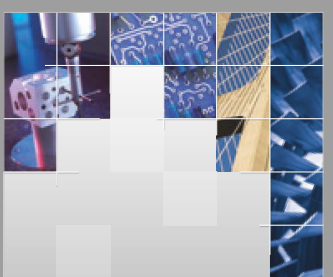

\section{Enfincering}
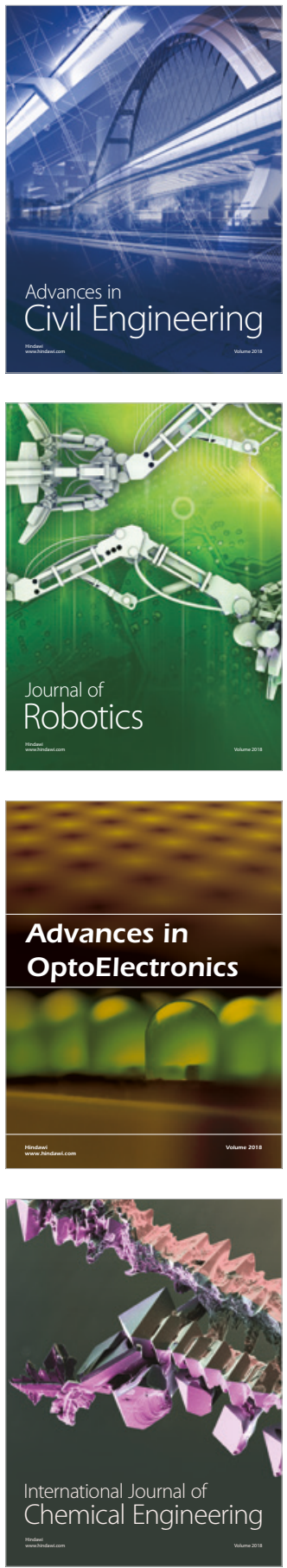

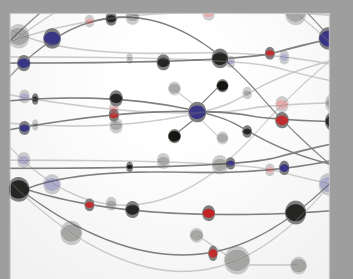

\section{Rotating \\ Machinery}

The Scientific World Journal

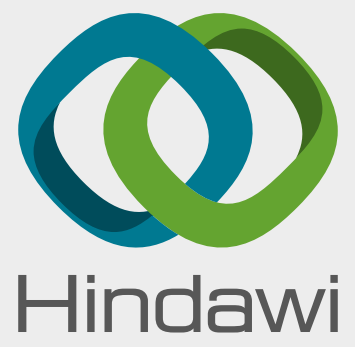

Submit your manuscripts at

www.hindawi.com
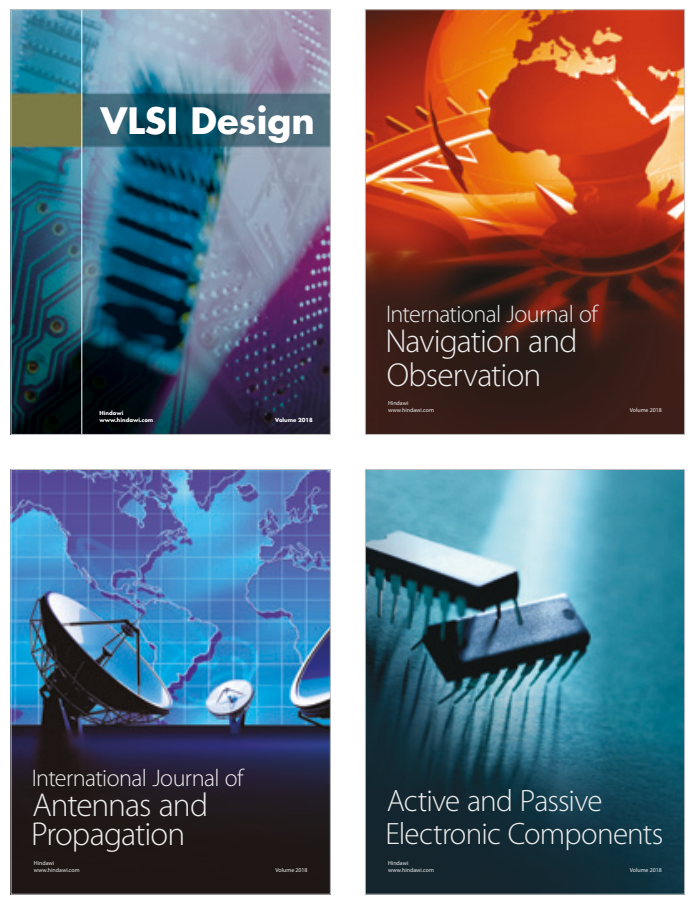
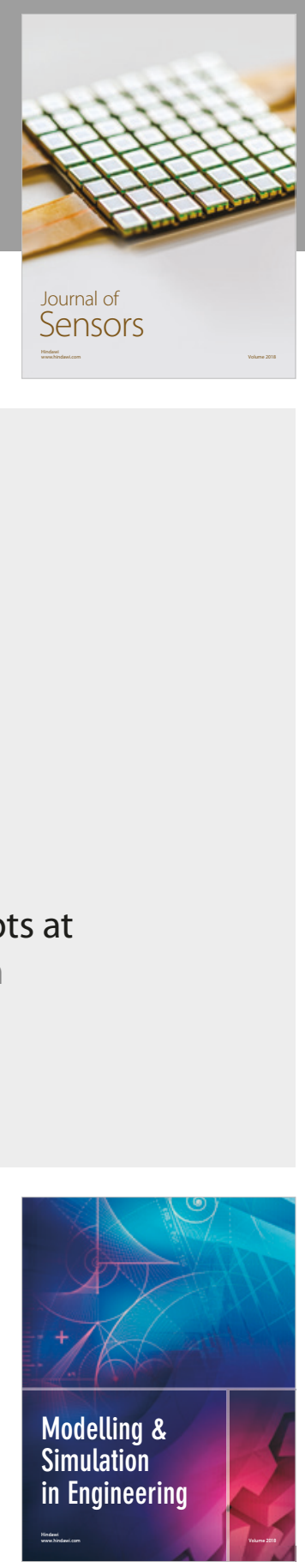

\section{Advances \\ Multimedia}
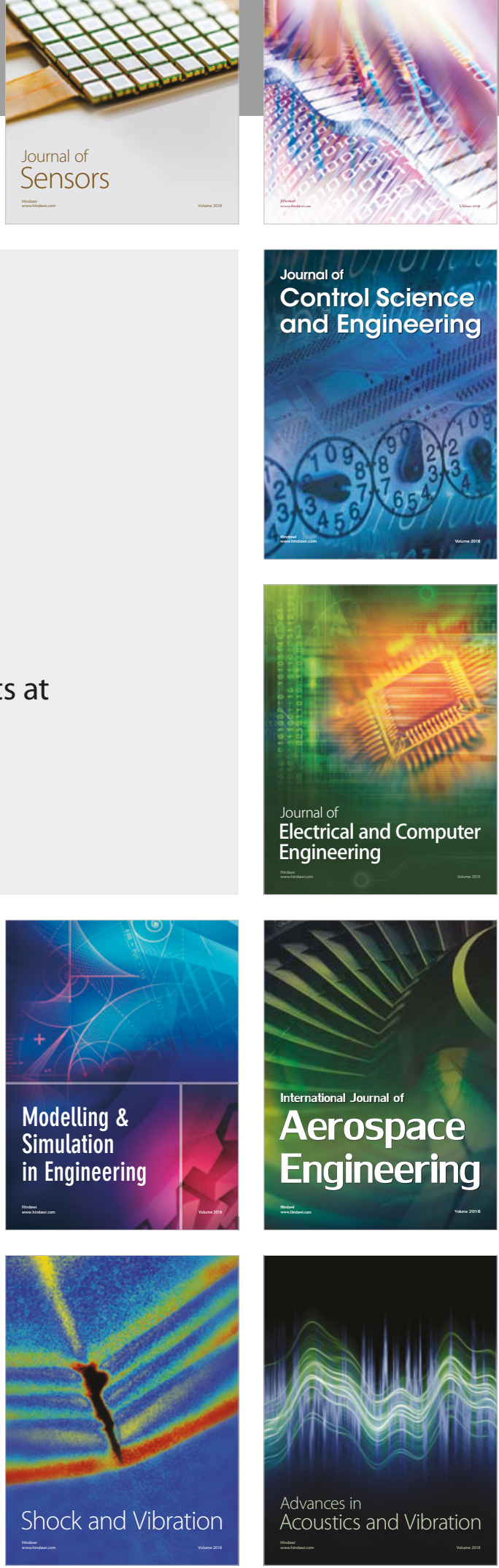